ESJ Social Sciences

\title{
Construction Et Première Validation D’un Mini- Questionnaire d'Inventaire Des Stratégies Identitaires Chez \\ Des Adolescents Migrants Scolarisés En Situation Transculturelle
}

\author{
Tamo Fogue Yannick \\ Doctorant, Psychologie du développement, Université de Yaoundé 1, \\ Cameroun \\ Tcheundjio Rosaline \\ Assistante, Psychologie cognitive et du développement, Université de \\ Yaoundé 1, Cameroun \\ Messanga Gustave Adolphe \\ Maître de Conférences, Psychologie sociale et expérimentale, Université de \\ Dschang, Cameroun \\ Nkelzok Komtsindi Valère \\ Professeur, Psychologie cognitive et du développement, Université de \\ Douala, Cameroun \\ Tsala Tsala Jacques Philippe Benoit \\ Professeur, Psychologie clinique et du développement, Université de \\ Yaoundé 1, Cameroun
}

\section{Doi:10.19044/esj.2021.v17n15p217}

Submitted: 11 December 2020

Accepted: 27 April 2021

Published: 31 May 2021
Copyright 2021 Author(s)

Under Creative Commons BY-NC-ND

4.0 OPEN ACCESS

Cite As:

Tamo Fogue Y., Tcheundjio R., Messanga G.A., Nkelzok Komtsindi V. \& Tsala Tsala J.P.B. (2021). Construction Et Première Validation D'un Mini-Questionnaire d'Inventaire Des Stratégies Identitaires Chez Des Adolescents Migrants Scolarisés En Situation Transculturelle.

European Scientific Journal, ESJ, 17(15), 217. Doi:10.19044/esj.2021.v17n15p217

\section{Résumé}

En contexte africain, le développement de la personnalité est tributaire des niches socioculturels régissant la construction de l'identité individuelle, sociale et du concept de soi du sujet. Cependant, le changement socioculturel entraine une rupture entre l'identité de base engagée dans son milieu anthropomorphiste d'origine et celle assignée en milieu d'accueil. Pour résoudre ce conflit identitaire, l’on construit des mécanismes que la littérature 
range dans le concept de “stratégies identitaires". Mais jusqu'ici, elle ne propose pas d'outils permettant de mesurer clairement ce construit. C'est pourquoi dans cette recherche, l'on se propose de développer un instrument psychométrique standardisé, permettant d'évaluer quantitativement la construction des stratégies identitaires chez les adolescents migrants scolarisés en situation transculturelle. Il est organisé autour de quatre facettes/dimensions/sous-échelles relatives au Camouflage/Clandestinité/Redéfinition de soi, l'Arrangement/Affichage de soi, l'Evitement/Réparation de soi, et le Déni/Annulation de soi ou d'autrui. Après étalonnage/validation auprès de 368 adolescents migrants dont 184 réfugiés centrafricains et 184 déplacés internes Extrême-Nord/Nord camerounais, des deux sexes, âgés de 12 à 18 ans et scolarisés à l'EstCameroun, les analyses factorielles exploratoires et en composantes principales lui confèrent des qualités métrologiques fiables $\left(\alpha_{\text {items }}[.68, .93]\right.$; $\left.\alpha_{\text {QISISTglobal }}=.78\right)$. Ainsi, la validité/fidélité et la cohérence interne du questionnaire sont satisfaisantes.

Mots clés: Stratégie Identitaire, Adolescents Migrants, Situation Transculturelle, Réfugié, Déplacé Interne

\section{Construction And First Validation Of An Inventory Mini- Questionnaire Of Identity Strategies For Migrant Adolescents Schooled In A Trans/Cross-Cultural Situation}

Tamo Fogue Yannick,

PhD Candidate, Developmental Psychology, University of Yaoundé 1, Cameroon

Tcheundjio Rosaline,

Assistant Lecturer, Cognitive and Developmental Psychology, University of Yaoundé 1, Cameroon

Messanga Gustave Adolphe,

Associate Professor, Social and Experimental Psychology, University of

Dschang, Cameroon

Nkelzok Komtsindi Valère,

Professor, Cognitive and Developmental Psychology, University of Douala, Cameroon

Tsala Tsala Jacques Philippe Benoit,

Professor, Clinical and Developmental Psychology, University of Yaoundé 1, Cameroon 


\section{Abstract}

In the African context, the development of the personality depends on the socio-cultural niches that construct the individual/social identity and the self-concept of the subject. However, socio-cultural change is causing a gap between the basic identity engaged in its original anthropomorphic environment and that assigned in the host environment. To resolve this identity conflict, they construct mechanisms that the literature classifies under the concept of “identity strategies”'. But so far, it does not offer tools to clearly measure this construct. This is why in the present research they propose to develop a standardized psychometric instrument, making it possible to quantitatively assess the construction of identity strategies of migrant adolescents who school in a transcultural situation. It is organized around four facets/dimensions/subscales relating to Camouflage/Clandestinity/SelfRedefinition, Arrangement/Self-Display, Avoidance/Self-Repair and Denial/Cancellation of Self or Others. After calibration/validation with 368 migrant adolescents including 184 Central African refugees and 184 internally displaced persons from the Far North/North Cameroon, of both sexes, aged 12 to 18 years old, who school in the East region of Cameroon, the exploratory factor and components main characteristics analysis give it reliable metrological qualities ( $\alpha_{\text {items }}[.68, .93]$; $\alpha_{\text {QISISTglobal }}=.78$ ). Thus, the validity/reliability and internal consistency of the questionnaire are satisfactory.

Key Words: Identity Strategy, Migrant Adolescents, Transcultural Situation, Refugee, Internally Displaced Person

\section{Introduction}

En Afrique, le lien entre les contextes anthropologiques, leurs variations/changements et le développement de l'enfant/adolescent est une problématique qui nécessite une adresse heuristique rigoureuse; Car, la personnalité y semble tributaire des artefacts socioculturels qui déterminent significativement la construction de l'identité à la fois individuelle et groupale, ainsi que l'émergence/acquisition des compétences développementales. En effet, tout individu au cours de son développement en famille ou dans la société, intériorise des normes qui guident sa personnalité, ses orientations et ses aptitudes de base (Bonnet, 2010 ; Retschitzki, 2011). Cependant, les africains assignent les tâches culturelles séquentielles aux étapes de développement qu'ils reconnaissent. Ils perçoivent le développement comme un processus sociogénétique basé sur les croyances et les pratiques culturelles, guidant systématiquement la socialisation, l'éducation et les attentes requises pour chaque stade ontogénétique (Nsamenang, 2013). Dans cette logique, les transformations de l'individu étant le fruit de sa participation à la vie sociétale 
(Latoki, 2010; Rizzi, 2014), les activités culturelles organisent mieux ses mutations évolutives via l'implication de soi dans des pratiques traditionnelles, considérées comme des pivots transitionnels du développement. Or débutant avec la puberté, l'adolescence marque l'entrée dans de nouveaux systèmes normatifs qui permettent au sujet de devenir agent de sa propre socialisation.

Par ailleurs, la construction de son identité et sa reconnaissance sociale progressive apparaissent comme les principaux enjeux de cette période. Mais face aux changements socioculturels imposés par les migrations, l'adolescent réfugié ou déplacé interne est confronté à la prise de conscience de sa dépendance affective et au nécessaire mouvement d'autonomie. Car, relativement forcé de se resocialiser sur fond de participation aux activités de la culture d'accueil, "même s'il réussi à se forger une identité équilibrée (achevée), une crise éventuelle peut surgir et perturber l'équilibre, en exigeant la mise en place de nouveaux repères identitaires... » (Maguiabou Tchidjo, 2015 : 126). En effet, devant se construire une double identité liée à la culture d'origine et à celle d'accueil, il fait face à une difficulté à concilier ces valeurs culturelles imposées par son éducation de base et les exigences liées au milieu d'accueil auquel il doit s'ajuster pour vivre en harmonie. D'où des conflits identitaires, car l'adolescent migrant se sent tiraillé entre la culture de son groupe ethnique d'origine/d'appartenance et un mode de vie transculturel qui retentissent différemment sur son adaptation sociale. On note ici un clivage entre un schéma (identité) collectif où l'adolescent migrant, qu'il soit réfugié ou déplacé interne, doit partager les mêmes aspirations/croyances/rituels et parfois la même apparence physique que ses pairs du milieu d'accueil. Or si " trop de séparation mène à la désaffiliation, trop de ressemblance mène à la désindividualisation» (Moro \& Barou, 2003 : 9).

L'on s'aperçoit que lorsqu'on adresse la problématique du développement psychosocial et affectif en situation de variation culturelle, les conflits socio-identitaires requièrent l'essentielle des attentions. Car quand on change de milieu socioculturel, on traine avec soi une identité de base qui a émergé de ce milieu anthropomorphiste et de ses niches développementales, en façonnant les premiers schémas cognitivo-comportementaux du sujet. Mais face aux attentes/exigences du milieu d'accueil, émerge une identité assignée qui crée des conflits et induit des inconforts socio-émotionnels. D’où l'intérêt pour la recherche de comprendre les mécanismes qui président à la construction des stratégies identitaires visant à s'y adapter. Paradoxalement, la quasi-absence des outils permettant d'en faire une évaluation objective constitue l'un des principaux obstacles à l'étude rigoureuse de ce construit qui est pourtant une compétence développementale observable/mesurable. La présente étude objecte de s'inscrire dans la démarche de Churchill (1979) pour y proposer une alternative. 


\section{Les stratégies identitaires dans le modèle socioculturel de Bajoit (2000)}

La notion d'identité est polysémique et se définit en fonction de la discipline dans laquelle elle s'inscrit. Pour ce qui est des sciences humaines, elle s'organise autour d'une identité sociale impliquant le fait d'être soi-même tout en étant semblable aux autres, "oscillant entre l'altérité radicale et la similarité totale» (Bajoit, 2000: 66); et d'une identité du moi/individuelle/personnelle qui est une acceptation simultanée de soi et des caractéristiques de son environnement d'appartenance, sous l'effet des forces sociales et culturelles (Erikson, 1972 ; Héroux, 2016). Quant aux stratégies identitaires, ce sont des actions psychologiques que l'individu mobilise pour atteindre un ou plusieurs buts socio-adaptatifs. Elles s'orientent en fonction de l'interaction et du contexte dans lequel elles se produisent (Camilleri, 1990, cité par Gutnik, 2002 : 121). Dans cette logique interactionniste, «la notion de stratégie se situe à l'articulation du système social et de l'individu, du social et du psychologique ; elle permet de lire, dans les comportements individuels ou collectifs, les diverses manières dont les acteurs "font avec" les déterminants sociaux, et en fonction de quels paramètres familiaux ou psychologiques ». Ainsi, les stratégies identitaires sont fonctions des contextes socioculturels et historico-psychologiques dans lesquels elles se produisent. Dans le contexte de refuge et de déplacement interne, leur construction et déploiement se justifient par le caractère potentiellement stigmatisant et parfois ethnocentrique des ressortissants du milieu d'accueil. Elles rendent compte de "la bataille que livre le sujet, soit pour trouver une réponse à l'attribution d'une identité stigmatisante, soit pour réduire l'écart possible entre l'identité pour soi et l'identité pour autrui » (Gutnik, 2002 : 9). On peut donc dire que les stratégies identitaires sont développées par les migrants dans un contexte socioculturel dans lequel ils doivent se reconstruire identitairement en tant que des individus qui ressemblent à ceux originaires du dit contexte, alors qu'ils diffèrent d'eux (Poujol, 2009).

D’après le modèle identitaire de Bajoit (2000) qui s'inscrit dans une perspective socioconstructiviste, l'identité fonctionnel du sujet dans toute situation socio-existentielle est la somme des identités sociale et du moi, ainsi que du concept de soi. Car ils se construisent au cours des différentes interactions sociales en fonction des contextes dans lesquelles ces interactions s'inscrivent. Ainsi, l'identité est une construction sociale et psychique qui s'inscrit dans un long processus au cours duquel des réaménagements doivent être faits en termes de (ré) conciliation identitaire entre les différentes composantes, à savoir : l'identité désirée qui renvoie à ce que l'individu voudrait être ; l'identité engagée qui renvoie à ce qu'il est et a été ; et l'identité assignée qui renvoie à ce qu'il croit que les autres voudraient qu'il soit. L'effort que l'individu fait pour concilier ces trois identités fait partie de ce que cet auteur appelle "le travail du sujet" ou "la gestion relationnelle de 
soi'”. Cet effort consiste à concilier son identité engagée et son identité assignée, c'est-à-dire, se faire reconnaître par les autres pour ce qu'on est et a été ; concilier son identité engagée et son identité désirée, c'est-à-dire, se reconnaître soi-même pour ce qu'on est et a été ; concilier son identité désirée et son identité assignée, c'est-à-dire, ce qu'on attend avec ce qu'on croit que les autres attendent de soi.

\section{Les modalités des stratégies identitaires dans le modèle de Mellini (2009)}

Des modifications majeures au modèle de Bajoit (2000) ont été faites par Mellini (2009) qui l'a structuré en paliers. En inscrivant l'identité dans une perspective socioculturelle, il l'organise autour de : l'identité ressentie basée sur une attirance physique et affective vers une culture désirée ou pas ; l'identité engagée pour soi en tant que membre d’une société/culture d'accueil, et pour les autres qui constituent son entourage culturel d'origine. Il dégage également un pallier objectif caractérisé par une identité assignée qui rend compte de ce que l'individu pense que les autres attendent de lui, souhaitent pour lui, ou lui attribuent. Entre ces différentes composantes identitaires, peuvent surgir des «tensions » intra-orientées qui concernent l'individu dans son rapport à soi-même (palier subjectif de l'identité), et extra-orientées qui concernent l'individu dans son rapport aux autres (palier objectif de l'identité). La résolution de ces tensions intrapsychiques nécessite la mise en place des stratégies de réaménagements dites identitaires qui sont fonctions des individus et de leur contexte socioculturel. En effet, l'identité se construit sous l'influence de plusieurs facteurs qui sont d'ordre biologique, psychologique et socioculturel (Retschitzki, 2011). Les facteurs psychologiques d'après Marchand (2009), renvoient à l'image que l'individu se fait de lui-même, à ses croyances, aux représentations de soi qui constituent une structure psychologique lui permettant de sélectionner ses actions et ses relations sociales. En ce qui concerne les facteurs culturels, Rizzi (2014 : 33) montre que la culture est dispensatrice d'une identité à la fois individuelle et groupale où "l'individu intériorise les modèles culturels qui lui sont imposés si bien qu'il s'identifiera à son groupe d'origine dès sa naissance ». Mais comment un adolescent migrant qui vit dans un milieu relativement différent de sa culture d'origine, peut rester différent tout en cherchant la reconnaissance des autres dans un contexte socioculturel qui lui assigne une identité sans tenir compte de ses aspirations personnelles ? Pour gérer les effets socioémotionnels de ces tensions identitaires, le sujet va mettre en place des mécanismes adaptatifs divers. Le but premier étant d'affirmer et défendre son identité hors-contrat qui reste à reconstruire malgré l'ethnocentrisme vécue dans le milieu d'accueil.

Dans le modèle de Mellini (2009), les stratégies identitaires qui conceptualisent ces mécanismes adaptatifs se déclinent en quatre composantes 
à savoir : la "clandestinité" basée sur une acceptation de l'identité transculturelle engagée pour soi, mais pas pour les autres, et d'un camouflage par affichage factuel de soi comme de sa culture d'origine lorsqu'on est en présence des siens ; l' “arrangement”' basé sur l'engagement à devenir membre de la culture d'accueil pour les " autrui significatifs » et le dévoilement de son identité transculturelle en tenant compte de la proximité avec les sujets semblables et ceux différents qui l'entourent (les siens) ; l' "affichage"' basé sur un dévoilement plus étendu et sans restriction de son identité transculturelle sur "le mode de la revendication fière ou de la normalisation discrète " (Op.cit.: 19), et l'adoption du style de vie transculturel sur fond d'identification complète de soi à la culture d'accueil et d'hostilité à l'endroit des membres de sa culture de provenance ; le “déni”, basé sur la méconnaissance de son obligation de vivre dans la culture d'accueil. Cette dernière stratégie se manifeste sous différentes formes à savoir : l'évitement, la redéfinition, la réparation et l'annulation de soi. L'évitement est un mode de résolution par formation réactionnelle, qui peut prendre des formes différentes. Toutes ces formes impliquent la dénégation ou le refus d'accepter son statut de réfugié ou de déplacé interne. Un individu qui adopte cette stratégie évite tout contact avec les personnes de la culture d'accueil. Il interagit exclusivement avec les personnes de sa culture d'origine tant dans ses relations occasionnelles que stables. La redéfinition consiste à vivre l'identité transculturelle qu'il intègre de façon progressive à son identité individuelle en construction. La réparation consiste à chercher les voies et moyens pour rentrer dans sa culture d'origine. L'annulation de soi se décline en plusieurs composantes à savoir : les idéations suicidaires, les tentatives de suicide, le suicide, la consommation de substances psychoactives. Elle apparait comme une stratégie radicale pour se libérer des contrats narcissiques et des alliances inconscientes qu'impose à l'individu, une culture au détriment de ses aspirations culturelles de base.

\section{Littérature psychométrique sur l'identité : Un bref état de la question}

La littérature propose des instruments permettant d'évaluer l'identité chez l'individu. Cependant, ils présentent des insuffisances psychométriques majeures en matière dévaluation des stratégies identitaires. En effet, Marsh (1990) a construit le Self-Description Questionnaire (SDQ) codé sur une échelle de type Likert à 6 point $(1=$ false $; \ldots ; 6=$ true $)$, pour mesurer l'identité sociale, individuelle et le concept de soi chez les adolescents âgés de 13 à 17 ans. Mais il apparait plus comme un rapport subjectif constitué de 102 items organisés autours de 11 sous-échelles, rendant comte de ce construit dans trois domaines. Ces domaines prennent néanmoins en compte quelques aspects socio-identitaires à savoir : académique (verbal et scolaire); non académique (habiletés physiques, apparence physique, relations avec les pairs 
du sexe opposé, relations avec les pairs de même sexe, honnêteté/confiance et stabilité socio-émotionnelle); général (habitudes, goûts, préférences culturels). Mais, bien qu'ayant fait l'objet de nombreuses analyses psychométriques qui supportent la sensibilité, la fidélité et la validité, avec une cohérence interne acceptable (alpha de Cronbach $(\alpha)=.88$ ), cette échelle ne permet pas une évaluation psychométrique directe de la construction des stratégies identitaires.

Par ailleurs, en s'inspirant du modèle des tâches développementale à l'adolescence de Damon (1983) et du SDQ, Emond (1996) a conçu un questionnaire constitué de cinq sous-échelles correspondant à ces tâches à savoir : la sous-échelle d'amitié, mesurant le support émotionnel/intimité et la loyauté/confiance; la sous-échelle de sexualité, mesurant la conscience/satisfaction/épanouissement sexuelle ; la sous-échelle de séparation-individuation, mesurant l'autonomie/indépendance; la souséchelle de pensée sociopolitique, mesurant la moralité autonome/posconventionnelle; et la sous-échelle du concept de soi global, mesurant la valeur/satisfaction de soi et la confiance en soi. Cette dernière, bâtie sur le nom de Général Self Scale et constituée de 10 items. Elle mesure le concept de soi général du sujet comme acceptation de ses propres actions et sentiments de confort puis d'appartenance, associé à l'image de soi et sens général d'un bienêtre social. Ces items sont: Overall, I have a lot to be proud of (item1); Overall, I am good (item2); Most things I do well (item3); Nothing I do ever seems to turn out right (item4); Overall, most things I do turn out well (item5); I don't have much to be proud of (item 6); I can do things as well as most people (item 7); I feel that my life is not very useful (itm8); If I really try I can do almost anything I want to do (item 9); Overall, I'm a failure (Item 10) (Emond, 1996). Mais cette méthode semble plus évaluer l'estime de soi que l'identité proprement dite, et donc, ne mesure pas réellement la construction des stratégies identitaires, surtout lorsqu'on est en situation de migration ou de changement socioculturel. Ce qui remet en cause sa rigueur dans le respect non seulement des principes de théorisation suffisante du construit, mais surtout d'équité/standardisation et de qualité de mesure dans sa validation. Or le paradigme de Churchill (1979) relève la nécessité de théorisation du construit précis, d'exhaustivité des items, d’homogénéité et de représentativité dans la construction et la validation des instruments psychométriques. En effet, les outils relevés plus haut n'évaluer pas clairement ce construit, encore moins telle que appréhendé dans la présente recherche. Il est donc nécessaire de d'élaborer une mini-échelle qui permet de mesurer clairement tous ses aspectes. Ce faisant, l'on procède dans cette étude, au développement et à la première validation d'un instrument psychométrique standardisé, permettant d'évaluer quantitativement la construction des stratégies identitaires chez les adolescents migrants scolarisés en situation transculturelle. 


\section{Méthode \\ Participants}

Ont participé à la présente étude, 368 adolescents dont 184 réfugiés centrafricains et 184 déplacés internes Extrême-Nord/Nord camerounais. Ils vivent dans la Région de l'Est du Cameroun, précisément dans les Arrondissements de Mandjou, Bétaré-Oya et Garoua-Boulaï, Département du Lom-et-Djérem. Ils sont tous âgés de 12 à 18 ans, des deux sexes, scolarisés au moins jusqu'en fin de premier cycle du secondaire, sans antécédent psychiatrique ni médical. Ils sont totalement ou partiellement en situation transculturelle, car vivent dans des communautés culturellement différentes de leurs origines. En effet, le milieu d'accueil est de culture Baya divisée en 02 Cantons (Gbaguinda et Bodomo), alors que ces adolescents migrants viennes des communautés Mbororo, Foulbé et Haoussa (Catholic Relief Services [CRS], entretien avec une unité déclarante, 24/10/2018).

\section{Matériel et procédure}

La littérature ne propose pas d'outil psychométrique évaluant la construction des stratégies identitaires chez des adolescents migrants et scolarisés/vivant en contexte transculturel, qui présente des qualités métrologiques avérées. Or il en existe pour l'identité, l'estime de soi et le concept de soi. Dans la présente recherche, l'on y trouve une solution en construisant un mini-questionnaire standardisé, rendant compte de ce mécanisme socio-adaptatif, comme stratégie d'aménagement psychologique ou de résolution des conflits identitaires en situation de changement socioculturel. Il s'agit de pouvoir quantifier les stratégies que les adolescents migrants scolarisés construisent à la fois en tant que redéploiement de leurs identités individuelles et sociales dans leur vécu socio-émotionnel quotidien ; et comme conciliatrices des identités ressenties, désirées ou engagés, avec l'identité à eux assignée dans un milieu socioculturel différent de leurs cultures de base/d'origine.

L'élaboration de cette mini-échelle psychométrique a consisté tout d'abord à s'inspirer de la littérature portant sur la culture et le développement de l'identité pour définir/circonscrire clairement le domaine du construit ou de la variable théorique à évaluer. Par la suite, le modèle typologique des mécanismes de construction socio-identitaire de Mellini (2009) a permis d'identifier ses facettes/modalités/dimensions, puis générer un échantillon d'items sur leurs indicateurs, considérés ici comme facteurs ou composantes principaux des stratégies identitaires. Ainsi, autour des quatre (4) facettes, l'on a d'abord généré 15 items, dont cinq (5) pour la première, trois (3) pour la seconde, quatre (4) pour la troisième et trois (3) pour la quatrième. Considérés comme des sous-échelles de l'instrument, ces facettes sont respectivement, le camouflage/clandestinité/redéfinition de soi, l'arrangement/affichage de soi, 
l'évitement/réparation de soi, le déni/annulation de soi ou d'autrui. En suivant le paradigme de Churchill (1979), l'on a fait un test préalable auprès des personnes proches des unités d'analyse cibles de l'étude. Il s'agit notamment de 50 adolescents réfugiés centrafricains et 50 adolescents déplacés internes Extrêmes-Nord/Nord-camerounais vivant tous dans l'arrondissement de Bertoua $2^{\text {ième }}$ (Région de l'Est du Cameroun). Ceci visait à éprouver chaque item et relever s'il est compréhensible pour les participants. L'on a associé à cette phase de validation, l'avis d'un psychologue du développement expérimenté sur les questions de construction identitaire. Ce qui a donné de supprimer deux (2) items pour la première sous-échelle ("Je ne fait que semblant d'aimer ou de rester avec les gens d'ici, mais au fond je force seulement" et 'CC'est parce que je suis obligé que je fréquente avec ceux qu'on a trouvé ici, ils m'énervent et je fais avec eux’”) ; et un (1) item pour la troisième ("Je ne peux être un jour tranquille que si la guerre fini complètement et je me retrouve dans mon quartier d'où je viens"'), pour non fiabilité psychométrique. En effet, plus de 30\% des répondants n’ont pas donné un avis à ces énoncés. Ce qui a remis totalement en cause leur sensibilité.

A la suite de ce pré-test, une mini-échelle définitive baptisée "MiniQuestionnaire d'Inventaire des Stratégies Identitaires en Situation Transculturelle (QISIST)'” a été conçue, pour être remplie pendant une durée de cinq (5) à dix (10) minutes. Elle est constituée de 12 items codés sur échelle de type Likert à 5 points notamment : $1=$ Pas du tout vrai ; $2=$ Un peu vrai ; $3=$ Modérément vrai ; $4=$ Assez vrai ; $5=$ Très vrai. La tâche et la consigne principales étant de cocher pour chacun des items, la case correspondant au numéro de la réponse qui est la plus appropriée au participant. Ceci permet de mesurer sa conception de soi dans une dimension académique et nonacadémique, basée sur la confiance en soi et la satisfaction de soi. Ce miniquestionnaire évalue précisément la relation avec les pairs, l’intégration dans la communauté d'accueil, la perception des autres, la satisfaction par rapport à sa vie scolaire transculturelle, la relation avec sa culture d'origine et celle d'accueil. Il s'organise autour des quatre (4) principales facettes ou stratégies identitaires relevées ci-dessus comme sous-échelles du mini-questionnaire, évaluées chacune par trois (03) items.

La première sous-échelle est relative au Camouflage/Clandestinité/Redéfinition de soi (QISIST 1 : C/C/R). Elle évalue la tendance du sujet à faire semblant d'être de la culture d'accueil, adoptant ses habitudes vestimentaires/alimentaires et en cherchant à parler la langue locale juste pour être à l'aise dans ses activités quotidiennes, mais au fond n'accepte pas ces réalités. Son item 2 est “Quand je suis avec mes frères réfugiés/déplacés internes de chez nous je suis mieux, mais quand je mène mes activités je fais comme si j'étais d’ici'”. La deuxième sous-échelle concerne 
l'Arrangement/Affichage de soi (QISIST 2 : A/A). Elle évalue la tendance du sujet à se définir comme étant du milieu d'accueil, à s'afficher dans toute la configuration culturelle de ce milieu, cherchant activement la proximité avec les pairs qui y sont originaires. Son item 1 est "J'aime la façon et le style de vie des gens d'ici, et par tout ou je suis, je fais aussi comme eux'. La troisième sous-échelle correspond à l'Evitement/Réparation de soi (QISIST 3 : E/R). Elle évalue la tendance du sujet à manifester des comportements d'aversion de contacts avec ses pairs originaires du milieu d'accueil et à vouloir absolument rentrer chez eux. Son item 3 est "Je cherche comment retourner chez nous pour faire comme chez nous étant à l'aise". La quatrième souséchelle porte sur le Déni/Annulation de soi ou d'autrui (QISIST 4 : D/A). Elle évalue la tendance du sujet à refuser totalement qu'il est dans un milieu autre que son milieu d'origine, à développer des violences vis-à-vis de ceux de la culture d'accueil, avec des idéations suicidaires. Son item 1 est "Si je reste trop ici, je sens que je dois finir par me suicider, car je refuse de faire comme les gens d'ici et je ne supporte plus d'être loin de chez nous'”.

Pour sa cotation, les scores sont obtenus et analysés en comptabilisant le score $(1,2,3,4$ ou 5) à chacun des items de Camouflage/Clandestinité/Redéfinition de soi $(\operatorname{Min}=0 ;$ Moy $=7,5 ; \operatorname{Max}=$ 15), d'Arrangement/Affichage de soi (Min $=0 ;$ Moy = 7,5; Max =15), d'Evitement/Réparation de soi (Min $=0$; Moy $=7,5 ; \operatorname{Max}=15)$ et de Déni/Annulation de soi ou d'autrui (Min = 0; Moy = 7,5; Max =15). Un résultat élevé indique selon les cas que l'individu développe majoritairement une des stratégies identitaires. Ainsi, pour chaque sous-échelle, l'on doit calculer l'écart entre le score du participant et son score moyen (Écart-Moyen (EM). La ou les sous-échelle (s) où l'Écart-Moyen est plus élevé indique la ou les stratégie (s) identitaire (s) la ou les plus régulièrement construite (s) par l'individu.

Pour sélectionner les 368 participants, un questionnaire d'identification a été d'abord administré pour recueillir des informations biographiques sur chaque participant, son lien ou sa vie avec sa famille (parents, fratrie et collatéraux) et ces antécédents médicaux, cliniques ou psychiatriques. Ainsi un test sélectif de stabilisation/homogénéisation/standardisation a été passé notamment, la version traduite par Weiss et Marmar (1997) de l'Impact of Event Scale (IES), composée de quinze (15) items évaluant l'Intrusion dérangeante (Items 1, 4, $5,6,10,11,14$ ) et l'Evitement (Items 2, 3, 7, 8, 9, 12, 13, 15) et réétalonnée/adaptée avec un alpha de Cronbach global $(\alpha)=.91$ et une fidélité $\left(r_{\text {test-re-test })}\right)=.62$. Cet exercice a permis d'inclure uniquement ceux qui ne présentent que des symptômes légers/mineurs de l'Etat de Stress Posttraumatique (ESPT) ou n'en manifestent pas du tout, vu leur entrave au développement socio-affectif (Zerach \& Solomon, 2018); et ceux n’ayant 
jamais présenté ou ne présentant pas de symptômes de carence alimentaire, vu leurs retentissements psychiques (affectif et cognitif) (Barberger-Gateau \& Berr, 2004). Au demeurant, ceci a favorisé une nouvelle collecte des données comme le stipule l'avant dernière étape du paradigme classique de Churchill (1979), suivi d'un étalonnage et de l'estimation via une analyse statistique rigoureuse, des qualités métrologiques de l’instrument.

\section{Résultats}

La validation d'un instrument de mesure est principalement fondée sur la satisfaction que procurent ses qualités métrologiques. Ainsi, en s'appuyant sur les usages de l'esprit psychométrique (Messanga, Kenne Tiotsop, \& Nzeuta Lontio, 2020 ; Messanga \& Nzeuta Lontio, 2020), l'on fournit dans les résultats de la présente étude les paramètres métriques sur la structure interne du Mini-Questionnaire d'Inventaire des Stratégies Identitaires en Situation Transculturelle. Tout d'abord, l'analyse factorielle exploratoire (AFE) et en composantes principales (ACP) a rendu compte de la qualité d'échantillonnage, de la saturation entre ses dimensions/composantes et du degré de mesure réelle du construit évalué. Puis, la statistique descriptive et les indices de corrélation test-ré-test ont favorisé l'estimation de sa fiabilité psychométrique (fidélité, sensibilité/validité/cohérence interne). Ensuite, les indices d'association linéaires des sous-échelles et l'analyse de la variance des scores ont montré ses liaisons inter-dimensionnelles, ainsi que sa validité discriminante. Enfin, les matrices des points-variables présentées sur les graphiques/cartes factorielles ont permis de décrire sa structure ou son homogénéité interne et celle de ses sous-échelles.

\section{Analyse factorielle exploratoire, variances expliquées et axes d'inertie des composantes principales}

Tableau 1. Matrice de présentation des indices de KMO, du test de sphéricité de Bartlett et des valeurs propres initiales des composantes (CP) principales du QISIS

\begin{tabular}{|c|c|c|c|c|c|c|c|c|}
\hline & \multicolumn{4}{|c|}{$\begin{array}{l}\text { Indices de KMO et test de sphéricité de } \\
\text { Bartlett }\end{array}$} & \multicolumn{4}{|c|}{ Valeurs propres initiales $(\lambda \alpha)$} \\
\hline & $\begin{array}{l}\text { Indice } \\
\text { KMO }\end{array}$ & $\begin{array}{ll}\text { Khi } & 2 \\
\text { approx. }\end{array}$ & Ddl. & $\begin{array}{l}\text { Sig. } \\
p\end{array}$ & $\mathbf{C P}$ & Total & $\begin{array}{l}\text { \% } \\
\text { variance }\end{array}$ & \% cumulé \\
\hline \multirow{3}{*}{$\begin{array}{l}\text { Echelle globale } \\
\text { (QISIST) }\end{array}$} & \multirow[t]{3}{*}{.812} & \multirow[t]{3}{*}{858.338} & \multirow[t]{3}{*}{66} & \multirow[t]{3}{*}{.000} & 1 & 3.456 & 28.803 & 28.803 \\
\hline & & & & & 2 & 1.673 & 13.938 & 42.741 \\
\hline & & & & & 3 & 1.018 & 8.483 & 51.224 \\
\hline $\mathrm{QISIST}_{1}(\mathrm{C} / \mathrm{C} / \mathrm{R})$ & .734 & 96.469 & 3 & .000 & 1 & 1.614 & 53.784 & 53.784 \\
\hline $\mathrm{QISIST}_{2}(\mathrm{~A} / \mathrm{A})$ & .727 & 81.417 & 3 & .000 & 1 & 1.530 & 51.000 & 51.000 \\
\hline $\mathrm{QISIST}_{3}$ (E/R) & .759 & 71.365 & 3 & .000 & 1 & 1.523 & 50.758 & 50.758 \\
\hline $\mathrm{QISIST}_{4}$ (D/A) & .650 & 199.032 & 3 & .000 & 1 & 1.847 & 61.564 & 61.564 \\
\hline
\end{tabular}


Notes: QISIST = Mini-Questionnaire d'Inventaire des Stratégies Identitaires en Situation Transculturelle ; QISIST $_{1}(\mathrm{C} / \mathrm{C} / \mathrm{R})=$ Dimension Camouflage/Clandestinité/Redéfinition de soi ; $\operatorname{QISIST}_{2}(\mathrm{~A} / \mathrm{A})=$ Dimension Arrangement/Affichage de soi ; $\mathrm{QISIST}_{3}(\mathrm{E} / \mathrm{R})=$ Dimension Evitement/Réparation de soi ; $\operatorname{QISIST}_{4}(\mathrm{D} / \mathrm{A})=$ Dimension Déni/Annulation de soi ou autrui ; $\mathrm{CP}=$ Composante principale.

\section{Source : Données de terrain de Janvier à Février 2020}

Les indices de Kaiser-Meyer-Olkin (KMO) et le test de sphéricité de Bartlett sont assez significatifs pour favoriser l'analyse factorielle sur les items du Mini-Questionnaire d’Inventaire des Stratégies Identitaires en Situation Transculturelle (QISIST). Ainsi, à partir des données factorisées, ils attestent des liaisons positives fortement significatives entre ses dimensions/facettes, avec des indices d'adéquation ou d'échantillonnage de bonne qualité. Cependant, l'AFE et l'ACP rendent compte de 3 facteurs ou composantes principales pour le mini-questionnaire globale, 1 facteur pour chacune de ses dimensions, avec des valeurs initiales propres supérieure à 1 et des solutions factorielles satisfaisantes. En effet, tandis que le QISIST global peut recueillir $56.784 \%$ des informations sur les Stratégies Identitaires en Situation Transculturelle, le $\mathrm{QISIST}_{1}$ (C/C/R), le QISIST 2 (A/A), le $\mathrm{QISIST}_{3}(\mathrm{E} / \mathrm{R})$ et le $\mathrm{QISIST}_{4}$ (D/A) peuvent en recueillir respectivement $53.784 \%$ sur le Camouflage/Clandestinité/Redéfinition de soi, $51.000 \%$ sur l'Arrangement/Affichage de soi, 50.758\% sur l'Evitement/Réparation de soi et $61.564 \%$ sur le Déni/Annulation de soi ou d'autrui. Donc le miniquestionnaire élaboré fournit des informations riches en quantité et en qualité sur le construit qu'on évalue chez les adolescents migrants. Ce qui rend nécessaire la description de ses scores auprès des participants et l'estimation de sa fiabilité psychométrique.

\section{Statistique descriptive et la fiabilité psychométrique de la mini-échelle et de ses sous-échelles}

Tableau 2. Paramètres descriptifs des scores, fidélité et cohérence interne du QISIST

\begin{tabular}{|l|l|l|l|l|c|}
\hline & M/É-T & MS/ÉM & $\begin{array}{l}\text { Indice de } \\
\text { fidélité } \\
\left(\mathbf{r}_{\text {test-re-test }}\right)\end{array}$ & $\begin{array}{l}\text { Indice } \\
\text { Alpha } \\
(\boldsymbol{\alpha})\end{array}$ & $\begin{array}{l}\text { Variation d'Alpha par } \\
\text { item }\end{array}$ \\
\hline $\begin{array}{l}\text { Mini-Echelle } \\
\text { globale (QISIST) }\end{array}$ & $29.11 / 11.19$ & $30 /-0.89$ & .76 & .78 & {$[.68, .93]$} \\
\hline QISIST $_{1}$ (C/C/R) & $8.28 / 3.57$ & $7.5 / .78$ & .83 & .71 & $\mathrm{I}_{1}=.69 ; \mathrm{I}_{1}=.70 ; \mathrm{I}_{1}=.73$ \\
\hline QISIST $_{2}$ (A/A) & $8.06 / 4.27$ & $7.5 / .56$ & .61 & .77 & $\mathrm{I}_{1}=.74 ; \mathrm{I}_{1}=.89 ; \mathrm{I}_{1}=.68$ \\
\hline QISIST $_{3}$ (E/R) & $7.49 / 4.64$ & $7.5 /-0.01$ & .81 & .75 & $\mathrm{I}_{1}=.73 ; \mathrm{I}_{1}=.76 ; \mathrm{I}_{1}=.75$ \\
\hline QISIST $_{4}$ (D/A) & $5.30 / 2.99$ & $7.5 /-2.20$ & .77 & .87 & $\mathrm{I}_{1}=.78 ; \mathrm{I}_{1}=.83 ; \mathrm{I}_{1}=.93$ \\
\hline
\end{tabular}

Notes : M = Moyenne ; É-T = Écart-Type ; MS = Moyenne standard ; EM = Écart-Moyenne

Source : Données de terrain de Janvier à Février 2020

A partir des statistiques descriptives (Moyenne ; Écart-Type) obtenues dans les deux strates d'adolescents migrants (Réfugiés et Déplacés internes), 
on dégage une forte variation des scores au mini-questionnaire global et à ses différentes facettes/dimensions. En plus, les paramètres de dispersion (Moyenne standard ; Écart-Moyenne) indiquent que les participants construisent majoritairement le Camouflage/Clandestinité/Redéfinition de soi, suivi de l'Arrangement/Affichage de soi, puis de l'Evitement/Réparation de soi et très peu de Déni/Annulation de soi ou d'autrui, comme Stratégies Identitaires. Il importe de rappeler ici la norme de cotation énoncée plus haut : "La ou les sous-échelle (s) où l'Écart-Moyen est plus élevé indique la ou les stratégie (s) identitaire (s) la ou les plus régulièrement construite (s) par l'individu» (Voir Méthode, B, Parag. 5, Dernière phrase). Cependant, les indices de corrélation test-ré-test et d'alpha de Chronbach générés démontrent respectivement une fidélité et une cohérence interne significativement satisfaisantes, tant au niveau des items que des sous-échelles et du miniquestionnaire global. D'où l'utilité d'analyser l'association linéaire et la variation inter facettes/items de cet instrument.

\section{Liaison inter-dimensionnelle et relation inter/intra groupes du QISIST chez les réfugiés et les déplacés internes}

Tableau 3.Association linéaire de Pearson (r) et variation inter facettes/items du QISIST

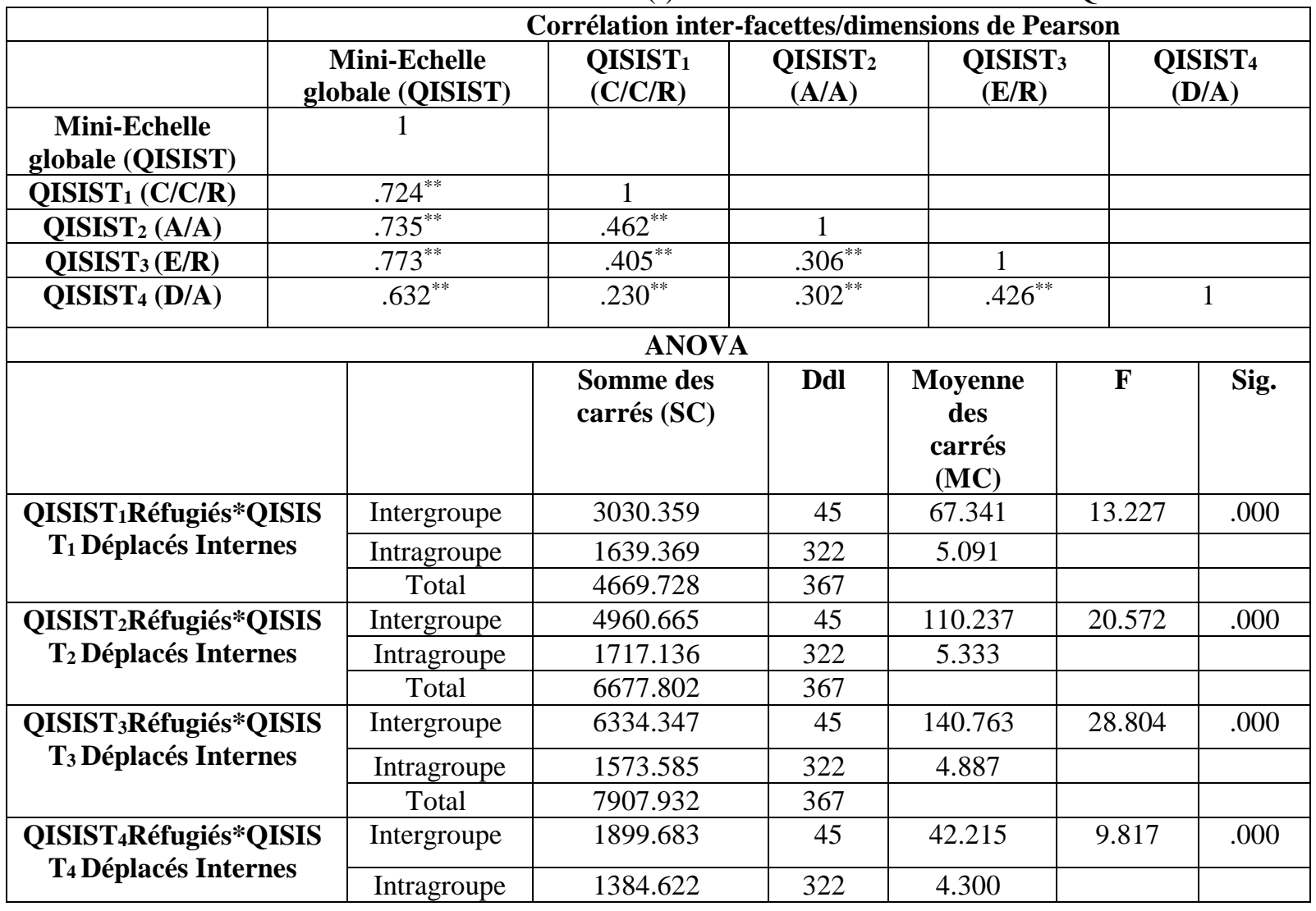




\section{Total}

3284.304

367

Notes: ${ }^{* *}$. La corrélation est significative au niveau .01 bilatéral. ANOVA $=$ Analyse de la variance ; Ddl = Degré de liberté ; F = Indice de Fischer Snedecor ; Sig.= Probabilité de validité (selon le seuil de signification).

\section{Source : Données de terrain de Janvier à Février 2020}

L'on note une de très fortes corrélations entre le mini-questionnaire global et ses sous-échelles. Ce qui démontre un lien positif et significatif entre les Stratégies Identitaires en Situation Transculturelle et les dimensions de ce construit. En observant les indices de corrélations entre les différentes souséchelles, l'on relève qu'il existe des liaisons positives entre ces dimensions. Cependant, prenant en compte les sous groupes d'âges et de sexe dans les deux strates des participants (réfugiés et déplacés internes), on note une variation globale significative des scores dans chaque dimension. Variation qui se montre plus significative/forte lorsqu'on passe d'un groupe à l'autre (intergroupe), qu'à l'intérieur d'un même groupe (intragroupe). De ce qui précède, l’on relève que la méthode psychométrique construite a des qualités métrologiques satisfaisantes. Toutefois, il semble utile de visualiser sa structure interne sur des représentations graphiques.

\section{Analyse de la structure interne du QISIST : Matrice des points-variables}
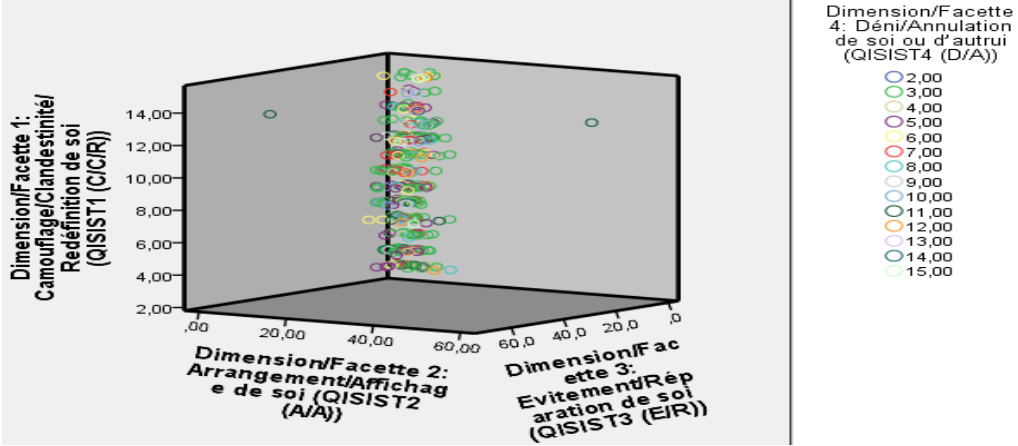

Figure 1. Carte/graphique factorielle du Mini-Questionnaire d'Inventaire des Stratégies Identitaires en Situation Transculturelle : QISIST

Source : Données de terrain de Janvier à Février 2020

A partir de la disposition des points-variables, l'on note un regroupement dans la partie centrale du graphique factoriel, avec une excentration d'un point du QISIST 1 à gauche et d'un autre du QISIST $_{3}$ à droite. Dans cette logique de rotation, la carte indique que les facteurs ou composantes principales du mini-questionnaire globale et de chacune de ses dimensions/facettes subit des changements certes, mais qui restent non significatifs dans la projection orthogonale des variables mesurées. Ainsi, l'on y relève une structure/homogénéité interne satisfaisante, avec un degré de 
dépendance inter-items qui corrobore à suffisance le niveau de corrélation élevé/significatif entre les dimensions/sous-échelles du questionnaire.

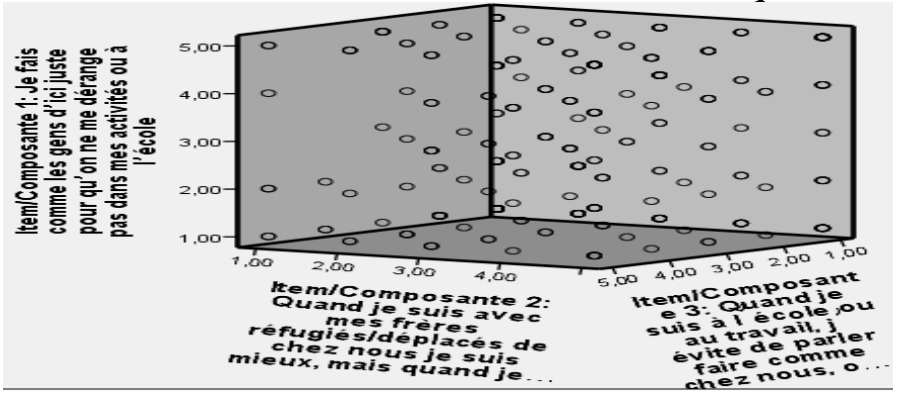

Figure 2. Carte/graphique factorielle des composantes de la dimension Camouflage/Clandestinité/Redéfinition de soi : $\mathrm{QISIST}_{1}(\mathrm{C} / \mathrm{C} / \mathrm{R})$

Source : Données de terrain de Janvier à Février 2020

Il ressort de cette carte factorielle, une dispersion significative des points-variables indiquant une faible saturation des scores aux items de la sous-échelle QIQIST 1 (C/C/R). Cette dispersion qui semble plus forte pour les items 2 et 3, montre que la distribution des biais sur cette dimension est faible, mais semble plus excentrique pour les deux derniers items qui constituent sa composante principale.

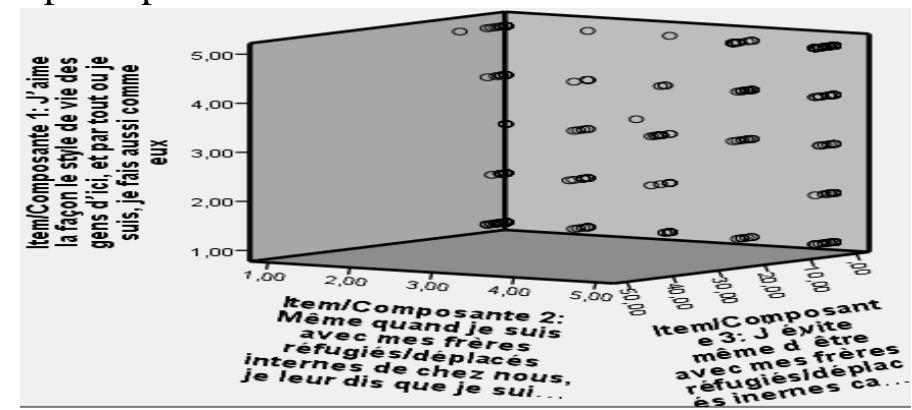

Figure 3. Carte/graphique factorielle des composantes de la dimension/sous-échelle Arrangement/Affichage de soi : Le QISIST 2 (A/A)

Source : Données de terrain de Janvier à Février 2020

Pour la dimension Arrangement/Affichage de soi, les points-variables des trois items sont concentrés dans la façade droite partant du centre du graphique factoriel. Ceci indique une forte convergence des variables de la sous-échelle QISIST $_{2}(\mathrm{~A} / \mathrm{A})$ vers une même composante principale portée par l'item 3. Cette disposition y confirme moins de changent factoriel, mais un degré d'homogénéité interne satisfaisant. 


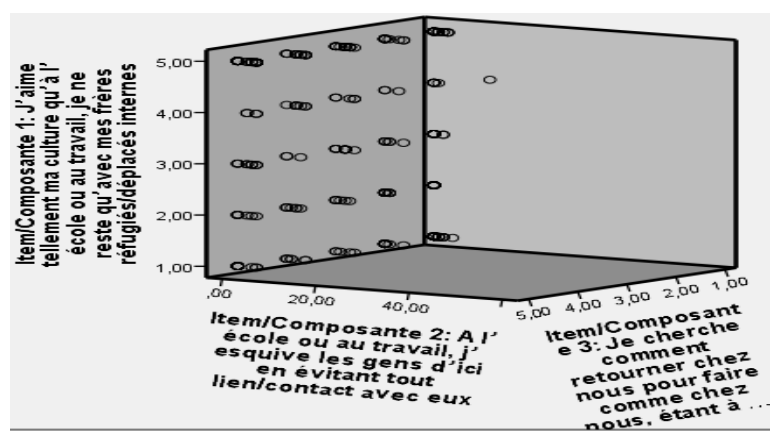

Figure 4. Carte/graphique factorielle des composantes de la dimension/sous-échelle Evitement/Réparation de soi : $\mathrm{QISIST}_{3}(\mathrm{E} / \mathrm{R})$

Source : Données de terrain de Janvier à Février 2020

Les variables de la sous-échelle $\mathrm{QISIST}_{3}(\mathrm{E} / \mathrm{R})$ convergent vers une même composante principale portée par l'item 1 dont les points sont concentrés dans la façade gauche, partant du centre de la carte factorielle. Ceci indique que la structure interne de la dimension Evitement/Réparation de soi a un niveau de saturation suffisant pour une bonne validité.

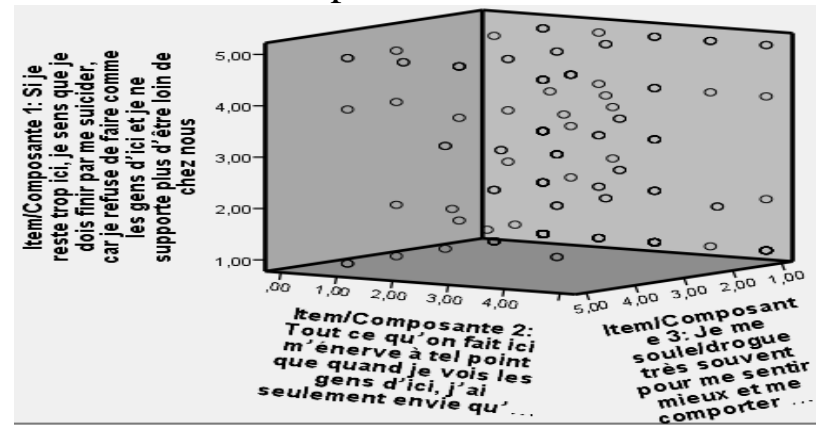

Figure 5. Carte/graphique factorielle des composantes de la dimension/sous-échelle

Déni/Annulation de soi ou d'autrui : $\mathrm{QISIST}_{4}$ (D/A)

Source : Données de terrain de Janvier à Février 2020

Pour la dimension Déni/Annulation de soi ou d'autrui, les pointsvariables indiquent une dispersion plus forte avec une distribution plus concentrique des biais non significatifs sur l'item 3. On note cependant quelques points des items 1 et 2 de cette sous-échelle qui convergent faiblement vers le centre du graphique factoriel. Ce qui implique que le $\mathrm{QIQIST}_{4}(\mathrm{C} / \mathrm{C} / \mathrm{R})$ a une composante principale portée conjointement par tous ses items avec une proportion élevée sur le dernier.

\section{Discussion}


Développer et valider un instrument psychométrique standardisé permettant d'évaluer quantitativement la construction des stratégies identitaires chez les adolescents migrants scolarisés en situation transculturelle, constituaient la mission principale de cette recherche. Ce faisant, les étapes du paradigme classique de Churchill (1979) ont présidé au respect de la rigueur dans l'élaboration du Mini-Questionnaire d'Inventaire des Stratégies Identitaires en Situation Transculturelle (QISIST), organisé autour de quatre facettes/dimensions/sous-échelles notamment, le Camouflage/Clandestinité/Redéfinition de soi (QISIST 1: C/C/R), l'Arrangement/Affichage de soi (QISIST 2 : A/A), l'Evitement/Réparation de soi (QISIST $3:$ E/R) et le Déni/Annulation de soi ou d'autrui (QISIST 4 : $\mathrm{D} / \mathrm{A}$ ). Ces dimensions émanent d'un cadre théorique et d'une littérature spécialisée sur le construit à évaluer/mesurer, ainsi que ses modalités, facteurs ou composantes. Ce qui a donné de générer d'abord un échantillon d'items, puis procéder à un pré-test qui a favorisé leur réduction et reformulation, avant d'aboutir au mini-questionnaire définitif qui respecte les normes et principes standards régissant l'esprit/ingénierie psychométrique (Roulin, 2017 ; Tiwari, Patel, \& Kumar, 2017).

En effet, en observant les principes d'équité/standardisation entre les répondants, de quantification et de qualité de mesure (Tay \& Jebb, 2017), les indices métriques calculés sur cette échelle d’auto-évaluation (Ruiller, 2008) lui consacrent des propriétés et qualités métrologiques fiables et satisfaisantes. Ceci s'affirme tant au niveau de sa sensibilité, sa validité, sa fidélité et sa cohérence interne, que des analyses factorielles exploratoires et en composantes principales faites. Ce qui corrobore avec la littérature psychométrique qui définit le cadre normatifs en matière d'étalonnage et de validation des instruments de mesures (Bernaud, 2014). Car le QISIST global et ses sous-échelles donnent des résultats satisfaisants auprès d'un échantillon suffisamment représentatif d'adolescents migrants, différentié en sexes (141 filles (77 réfugiés et 64 déplacés internes), 227 garçons (107 réfugiés et 120 déplacés internes) ; en âges (34 de 12-14 ans (17 réfugiés et 17 déplacés internes), 334 de15-18 ans (167 réfugiés et 167 déplacés internes)), et en origine/provenance (187 réfugiés et 187 déplacés internes). Etant donné que les indices apportés par la méthode d'alpha de Cronbach, la vérification des poids et des structures factoriels avec les pourcentages d'inertie cumulés obtenus sur les facteurs retenus se situent au dessus des seuils standards (.60, .70 , etc.).

\section{Conclusion}

Au regard même de la structure interne du mini-questionnaire global et de ses sous-échelles, factorisée et visualisée sur les cartes/graphiques factorielles, vu le fait que la validité d'un questionnaire psychométrique est 
aussi tributaire de sa capacité à renseigner sur la variabilité inter et intra individuelle (Trochim, 2006), l'on peut affirmer que le Mini-Questionnaire d'Inventaire des Stratégies Identitaires en Situation Transculturelle (QISIST) construit et validé dans cette recherche est fiable du point de vue psychométrique. À cet effet, l'on propose de l'adopter comme outil métrique, permettant d'évaluer quantitativement la construction des stratégies identitaires chez les adolescents migrants scolarisés en situation transculturelle, surtout en contexte Afrique francophone. Ceci en ouvrant la voie à d'autres investigations dans l'optique de son perfectionnement, de sa traduction et de son adaptation-réétalonnage. L'idéal étant d'augmenter sa légitimité scientifique pour un large spectre d'emploi dans l'étude de l'adaptation sociale en situation de changement culturel en Afrique.

\section{References:}

1. Bajoit, G. (2000). Notes sur la construction de l'identité personnelle. Recherches sociologiques, 2, 69-84.

2. Barberger-Gateau, P. \& Berr, C. (4-5 novembre 2004). Nutrition and cognitive decline: data from Paquid and Eva epidemiologic studies. Communication présentée au $4{ }^{\text {ème }}$ Congrès Européen sur la nutrition, la santé et le vieillissement, Toulouse.

3. Bernaud, J.-L. (2014). Méthodes de tests et questionnaires en psychologie. Paris : Dunod. En ligne : http://excerpts.numilog.com/books/9782100587957. Consulté $12 / 11 / 2020$.

4. Bonnet, D. (2010). La construction sociale de l'enfance : une vérité de normes et de contextes. Informations sociales. 4, 160, 12-18.

5. Churchill, G. A. (1979). A paradigm for developing better measures or marketing constructs. Journal of Marketing Research, 16(1) 64-73.

6. Damon, W. (1983). Social and personality development: Infancy through adolescence. New York: Cambridge University.

7. Emond, C. (1996). Relation entre tâches développementales, concept de soi et symptomatologie dépressive : étude transversale en milieu et fin de l'adolescence. Thèse doctorat. Canada : Ecole de psychologie, école des études supérieures de l'Université d'Ottawa.

8. Erikson, E. H. (1972). Adolescence et crise. La quête de l'identité. France : Flammarion.

9. [Gutnik, F. (2002). Autour des mots, "stratégies identitaires », « dynamiques identitaires ». Recherche et formation, 41, 119-130. En ligne :

https://www.persee.fr/doc/refor_09881824_2002_num_41_1_1778. Consulté le 18/03/2019. 
10. Héroux, A. T. (2016). La perturbation identitaire chez le trouble de la personnalité limite : Réflexion sur les aspects théoriques, empiriques et sur la mesure (Thèse de doctorat, Univerité du Québec, Québec). En ligne : http://depot-e.uqtr.ca/id/eprint/7863. Consulté le 15/05/2019.

11. Latoki, P-E. (2010). La religion comme quête de l'ordre dans la société africaine traditionnelle. Les cahiers psychologie politique, 16. En ligne : http://lodel.irevues.inist.fr/cahierspsychologiepolitiqu/index.php?id=14 15. Consulté le 28/09/2018.

12. Maguiabou Tchidjo, A. (2015). Grossesse précoce et construction de l'identité maternelle chez l'adolescente camerounaise. Thèse de doctorat en psychologie du développement. Cameroun : Université de Yaoundé I.

13. Marchand, R. (2009). Influences de la culture et de l'identité sur l'apprentissage du F.L.E. : Etude comparative des enseignements/apprentissages en France et en Chine. Thèse, de doctorat. France: Université de Lorraine. En ligne : www.theses.fr/2009NAN21026. Consulté le 14/02/2019.

14. Marsch, H. W. (1990). Self Description Questionnaire-II. Campbelltown, Australia :University of Western Sydney, Macarthur.

15. Mellini, L. (2009). Entre normalisation et hétéronormativité : la construction de l'identité homosexuelle. Déviance et Société, 1 (33), 326.

16. Messanga, G. A. \& Nzeuta Lontio, S. (2020). Construction et Validation d'une Échelle d'évaluation du Tribalisme dans les Relations Intergroupes. European Scientific Journal, 16, (10), 195-215. En ligne : http://dx.doi.org/10.19044/esj.2020.v16n10p195. Consulté 07/12/2020.

17. Messanga, G. A., Kenne Tiotsop, L. \& Nzeuta Lontio, S. (2020). Construction et Validation D'une Méthode D'évaluation D'évaluation Du Tribalisme Aversif. International Multilingual Journal of Science and Technology (IMJST), 5(11), 1896-1907. En ligne: http://imjst.org/index.php/vol-5-issue-11-November-2020/. C Consulté $5 / 12 / 2020$.

18. Moro, M. R. \& Barou, J. (2003). Les enfants de l'exil : Etude auprès des familles en demande d'asile dans les centres d'accueil. France: Sonacotra \& Unicef.

19. Nsamenang, A. B. (2013). Dilemmas of Rights-Based Approaches to ChildWell-Being in an African Cultural Context. In. D. J. Johson, D. L. Agbényiga, R. K. Hitchcock (Eds.), Vulnerable Children: Global Challenges in Education, 13 Health, Well Being, and Child Rights (PP. 13-25), New York: Hardcover. 
20. Paduart, P. (2004). Psychisme et culture. Revue Belge de Psychanalyse, 45. En ligne: http://www.gercpea.lu/textes_livres/psychisme et culture_P_Paduart.pdf. Consulté le 12/01/ 2019.

21. Poujol, J. (2009). L'accompagnement psychologique et spirituel. France : L’Empreinte ;

22. Retschitzki, J. (2011). La culture en tant que facteur du développement cognitif. Alterstice, 1(1), 81-84.

23. Rizzi, T. A. (2014). Entre ici et là-bas, je vous dessine mon chez moi : Exploration qualitative des productions des enfants en psychothérapie transculturelle. Thèse de doctorat. France : Université Paris Descartes. En ligne : https://tel.archives-ouvertes.fr/tel-01195999. Consulté le 28/01/2019.

24. Rogoff, B. (2003). The cultural nature of human development. Oxford, England: Oxford University Press.

25. Roulin, J. L. (2017). Savoir, Comprendre, Apprendre. Leçons de psychométrie. Université Savoie Mont Blanc. En ligne : https://www.psychometrie.jlroulin.fr. Consulté 24/11/2020.

26. Ruiller, C. (2008). Le soutien social au travail : conceptualisation, mesure et influence sur l'épuisement professionnel et l'implication organisationnelle : L'étude d'un cas hospitalier. Thèse de doctorat. France : Université Rennes 1.

27. Tay, L. \& Jebb, A. (2017). Scale Development. In S. Rogelberg (Éd.), The Sage Encyclopedia of Industrial and Organizational Psychology. Sage: Thousand Oaks, CA.

28. Tiwari, S. K., Patel, A.K., \& Kumar, D. (2017). Development of Perceived Discrimination Questionnaire: A measure for Different Social Categories Students. Journal of Psychosocial Research, 12, 1, 81-88.

29. Trochim, W. M. (2006). The Research Methods Knowledge Base. En ligne. http://www.socialresearchmethods.net/kb/. Consulté le 27/10/2020.

30. Tsala Tsala, J. Ph. (2006). La psychologie telle quelle perspective africaine. Yaoundé : PUCAC.

31. Weiss, D. \& Marmar, C.R. (1997). The Impact of Event Scale-Revised. In: J. Wilson \& T.M. Keane TM, Assessing psychological trauma and PTSD: A hand book for practitioners (399-411). New York: Guilford press.

32. Zerach, G. \& Solomon, Z. (2018). Gender differences in posttraumatic stress symptoms among former prisoners of wars' adult offspring. Anxiety, Stress, \& Coping, 31, 21-31. 


\section{Mini-Questionnaire d'Inventaire des Stratégies Identitaires en Situation Transculturelle (QISIST)}

Consigne : S'il vous plait, lisez chaque affirmation ci-dessous et dites à quel point elle est vraie pour vous. Veuillez indiquer en cochant le numéro de la réponse qui correspond le plus à votre avis. S'il vous plait soyez honnêtes. Vos réponses sont strictement confidentielles et ne seront utiles que si elles vous décrivent réellement

1= Pas du tout vrai ; $2=$ Un peu vrai ; 3= Modérément vrai ; 4= Assez vrai ; $5=$ Très vrai

\begin{tabular}{|c|c|c|c|c|c|c|}
\hline \multirow[t]{2}{*}{$\mathbf{N}^{\mathbf{0}}$} & \multirow[t]{2}{*}{ Items/composantes } & \multicolumn{5}{|c|}{$\begin{array}{l}\text { Cochez votre meilleur } \\
\text { choix }\end{array}$} \\
\hline & & 1 & 2 & 3 & 4 & 5 \\
\hline \multicolumn{7}{|c|}{ Camouflage/Clandestinité/Redéfinition de soi (QISIST $1:$ C/C/R) } \\
\hline 1 & $\begin{array}{l}\text { Je fais comme les gens d'ici juste pour qu'on ne me dérange pas dans mes activités } \\
\text { ou à l'école ; }\end{array}$ & 1 & 2 & 3 & 4 & 5 \\
\hline 2 & $\begin{array}{l}\text { Quand je suis avec mes frères réfugiés/déplacés internes de chez nous je suis mieux, } \\
\text { mais quand je mène mes activités je fais comme si j’étais d’ici }\end{array}$ & 1 & 2 & 3 & 4 & 5 \\
\hline 3 & $\begin{array}{l}\text { Quand je suis à l'école ou au travail, j'évite de parler et faire comme chez nous, ou } \\
\text { pour le faire, je me cache d'abord }\end{array}$ & 1 & 2 & 3 & 4 & 5 \\
\hline \multicolumn{7}{|c|}{ Arrangement/Affichage de soi (QISIST $2:$ A/A) } \\
\hline 1 & $\begin{array}{l}\text { J'aime la façon et le style de vie des gens d'ici, et par tout ou je suis, je fais aussi } \\
\text { comme eux }\end{array}$ & 1 & 2 & 3 & 4 & 5 \\
\hline 2 & $\begin{array}{l}\text { Même quand je suis avec mes frères réfugiés/déplacés internes de chez nous, je leur } \\
\text { dis que je suis d'ici et fiers de l'être }\end{array}$ & 1 & 2 & 3 & 4 & 5 \\
\hline $\begin{array}{l}3 \\
3\end{array}$ & $\begin{array}{l}\text { J'évite même d'être avec mes frères réfugiés/déplacés internes, car tout ce qu’on fait } \\
\text { chez nous m'énerve, je préfère faire comme ici, et demeurer ici }\end{array}$ & 1 & 2 & 3 & 4 & 5 \\
\hline \multicolumn{7}{|c|}{ Evitement/Réparation de soi (QISIST $3:$ E/R) } \\
\hline 1 & $\begin{array}{l}\text { J'aime tellement ma culture qu’à l'école ou au travail, je ne reste qu'avec mes frères } \\
\text { réfugiés/déplacés internes }\end{array}$ & 1 & 2 & 3 & 4 & 5 \\
\hline 2 & A l'école ou au travail, j’esquive les gens d'ici en évitant tout lien/contact avec eux & 1 & 2 & 3 & 4 & 5 \\
\hline 3 & Je cherche comment retourner chez nous pour faire comme chez nous étant à l'aise & 1 & 2 & 3 & 4 & 5 \\
\hline \multicolumn{7}{|c|}{ Déni/Annulation de soi ou d'autrui (QISIST $4:$ D/A) } \\
\hline 1 & $\begin{array}{l}\text { Si je reste trop ici, je sens que je dois finir par me suicider, car je refuse de faire } \\
\text { comme les gens d'ici et je ne supporte plus d'être loin de chez nous }\end{array}$ & 1 & 2 & 3 & 4 & 5 \\
\hline 2 & $\begin{array}{l}\text { Tout ce qu'on fait ici m'énerve à tel point que quand je vois les gens d'ici, j'ai } \\
\text { seulement envie qu'ils disparaissent tous }\end{array}$ & 1 & 2 & 3 & 4 & 5 \\
\hline 3 & $\begin{array}{l}\text { Je me soule/drogue très souvent pour me sentir mieux et me comporter partout } \\
\text { comme chez nous, sans avoir peur }\end{array}$ & 1 & 2 & 3 & 4 & 5 \\
\hline
\end{tabular}

